researchers in three previously disparate areas of research (vascular biology, neuroscience and immunology) all stumbled on the same molecule.

Within a short period of time, it was realized that $\mathrm{NO}$ was involved in the regulation of blood vessels, in communication in the brain, and in immunological defence against invading organisms. Now, NO impinges on almost all areas of biology. Not only is it important physiologically as a messenger that conveys signals from one cell to another in many different tissues but, through its toxic effects, it is also a likely player in many pathological processes.

So it was decided that we need to have a journal devoted specifically to the molecule, Nitric Oxide: Biology and Chemistry. But do we? It could also be said that, because of its importance to so many areas of biology, a journal solely concerned with NO is a nonstarter: NO is really only interesting in context. After all, we don't have, or need, journals specifically about glutamate or acetylcholine or inositol trisphosphate.

Viewed from this perspective, there is no shortage of journals in which to publish and read about NO research. In fact, almost any one will do.

Nitric Oxide: Biology and Chemistry is a spin-off from Archives of Biochemistry and Biophysics and is also referred to as 'Part B' of that journal. The first issue appeared in February 1997 and, so far, only eight issues have been published (six of which were from last year) so it is possibly a little early to expect the journal to have found its flavour.

The editors would like it to be a repository of papers about all aspects of the biology and chemistry of NO, but this is probably unrealistic. To date, there have been a few reviews, and a modest number (up to ten) of research papers per issue (except the latest: volume two, number two, which is a book of abstracts). Some of the better papers have come from the editorial advisory board's research groups. These aside, the journal appears to be falling into its default guise, which is Archives of Biochemistry and Biophysics Part B. The parent journal has long had a penchant for chemically oriented research into reactive free radicals, and $\mathrm{NO}$ does have this chemical characteristic (although it is not very reactive).

Of course it is important that the chemistry of NO and related species, such as the much more reactive peroxynitrite anion, does become better understood. Indeed, it is just this type of research that one imagines could find a home in a journal devoted exclusively to NO. The problem is that, when the chemistry is not constrained within a biological framework, you can get free radicals to do almost anything.

John Garthwaite is at the Wolfson Institute for

Biomedical Research, University College London, 140 Tottenham Court Road, London W1P 9LN, UK.

\section{Venturing outside the laboratory}

\section{Field Analytical Chemistry and Technology \\ Editor Henk L. C. Meuzelaar \\ Wiley. 6/yr. USA \$195, elsewhere \$231 \\ Patrick MacCarthy}

As analytical chemistry has evolved, it has focused primarily on the determination of substances within the controlled confines of a fixed laboratory. While there has always been an interest in conducting analyses where a sample is actually found, such onsite measurements have generally been found impractical for the more complex determinations that require highly sophisticated instrumentation and methodology.

Nevertheless, there has been a constant effort to adapt even the most sophisticated methods for portable use in the field. Such efforts introduce the inevitable challenges of instrument ruggedness, availability of appropriate power supplies, and control of operating parameters.

Field measurement is necessary when samples need to be analysed at their site of occurrence, as, for example, when dealing with unstable analytes; when immediate results are required, such as in detecting chemical or microbiological warfare agents on the battlefield; and when the analyst cannot be present, such as for analyses performed on the surfaces of other planets.

Field Analytical Chemistry and Techno$\log y$ is the first journal to focus on this area, and fills an important niche. It is international and interdisciplinary, publishing refereed, original research papers, short technical notes, reviews and so-called 'Outfield Reports'. The latter articles arise from what is described as "major field analytical projects involving multidisciplinary teams, often operating under highly demanding conditions". Stimulating editorials add to the journal's appeal.

Although the editorial board is international, with members representing nine different countries, and the journal policy is international in scope, 75 per cent of the articles across three issues came from US laboratories.

The journal has a professional, highquality appearance and is nicely laid out. The period from submission to acceptance is generally less than two months; the lag for actual publication also appears to be short, but is difficult to discern exactly as copies of the journal are identified by volume and issue number within a given year rather than by month of publication.

Patrick MacCarthy is in the Department of Chemistry and Geochemistry, Colorado School of Mines, Golden, CO 80401, USA.

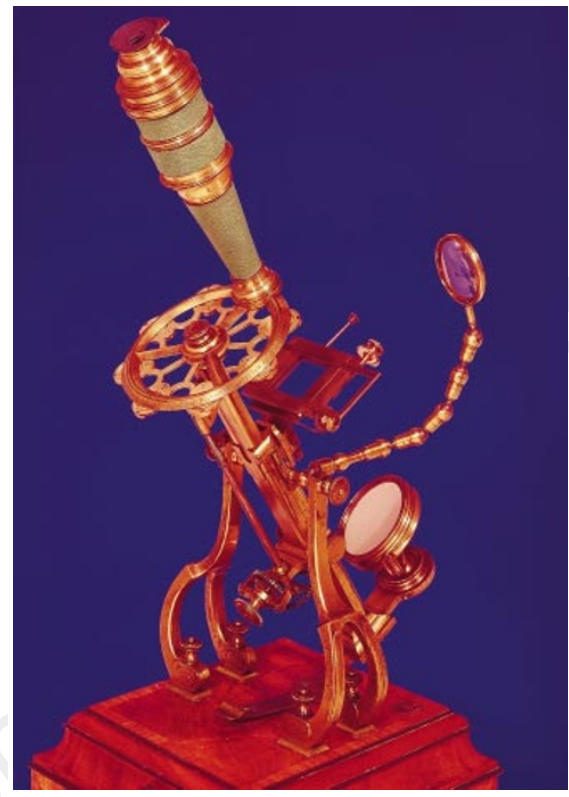

The Prince of Wales microscope, c. 1755.

\section{A site for sore eyes}

Microscopy and Microanalysis

Editor-in-chief Dale E. Johnson

Springer. 6/yr. \$539 (institutional)

\section{L.M.Brown}

Microscopes are to modern science what telescopes are to astronomy: a great enabling technology, which has brought about a considerable part of what is now undergraduate physics. Yet microscopy remains a discipline for professionals, and stands quite aloof from mainstream academic science, whether biological or physical.

Technical developments, often carrying in their train consequences of the utmost importance, such as new routes to the fabrication of devices on the nanometre scale, are announced either at specialized conferences or in the pages of Nature, but not usually in journals of physics or biology. So journals that cover conference programmes and details of trade exhibitions play a special role. They are sought after by every group that develops or applies microscopical techniques, especially when they concern structure and function of materials, whether biological or inorganic. The latest such publication is Microscopy and Microanalysis, the official journal of several major microscopical societies of North and South America. It is affiliated to the major European societies, and makes a fair bid to become the international house journal of microscopists.

It boasts an editorial board filled with eminent American pioneers of microscopy, nicely balanced between those concerned with biological problems and those concerned with the science of materials. North Americans predominate, but there is a smattering of Europeans and South Americans. 
The articles are handsomely printed on glossy paper, so that micrographs are excellently reproduced. Their content is typically a mixture of technique and application. About a quarter of each issue is taken up with book reviews, news and commentary, with advance information on a variety of conferences. All aspects of microscopy and microanalysis are covered, although electron microscopy dominates at the moment. There is a lively correspondence section.

Any institution outside America with research programmes in microscopy should have access to a copy of this journal. Active members of the North American community will want to become regular readers.

L. M. Brown is at the Cavendish Laboratory, University of Cambridge, Madingley Road, Cambridge CB3 OHE, UK.

\section{Digging up the recent past}

\section{International Journal of Historical Archaeology}

Editor Charles E. Orser Jr.

Plenum Press. 4/yr. USA \$100, elsewhere

$\$ 115$ (institutional); \$35 (personal)

\section{Frans Verhaeghe}

The archaeologies of the medieval, industrial and particularly early modern periods are relative newcomers, the latter being the most recent addition. Since the 1960s, it has been steadily growing, notably in the AngloSaxon world. Elsewhere in Europe, the importance and potential of sixteenth- to eighteenth-century remains are now also slowly being recognized. Journals such as Post-Medieval Archaeology and Historical

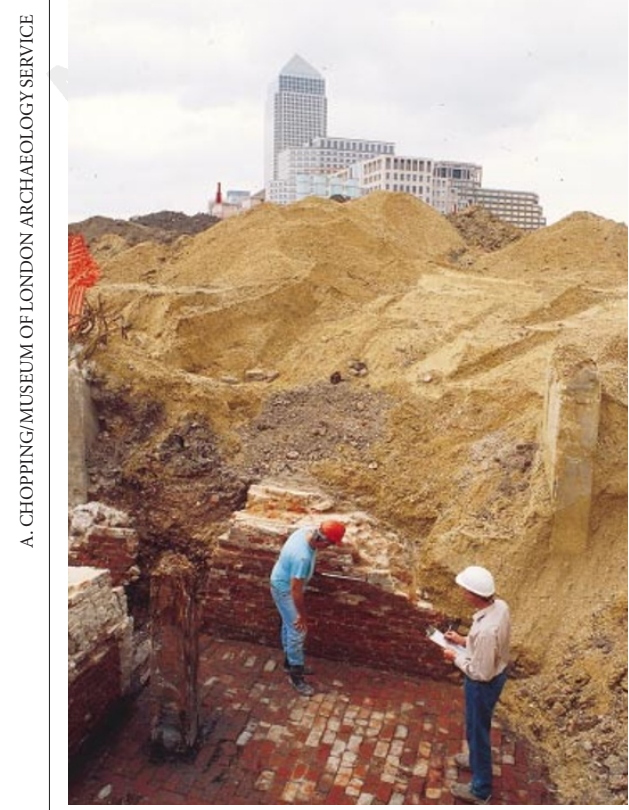

A dig at Lime Kiln Dock in London, overlooked by the tower of Canary Wharf.
Archaeology (published since 1967 by the UK Society for Post-Medieval Archaeology and the US Society for Historical Archaeology respectively) have created models, but there is room for more international approaches as well as more theoretical and applied research. Hence this new journal.

Judging from the editor's introduction, the journal's target area is the archaeology of any 'historical' period in any region of the planet. It offers medium-sized to more extensive papers, from about 10 to 45 pages long. Some are reprints of less readily accessible papers, but most of the 20 -odd papers now published are new, and all bar one focus on the past half-millennium and on the formerly colonized world outside Europe. The contributions are well presented and seem to be published fairly rapidly, in a handy $23 \mathrm{~cm}$ by $15 \mathrm{~cm}$ standard format, on paper allowing for good drawings and reasonable (though not high-quality) photographs.

A 'Views and Commentaries' section presents innovative and thought-provoking statements on issues confronting archaeologists of historical periods. Reviews and book reviews are not (yet?) offered but comments on published papers occasionally are.

The contents and scope of the first two volumes are very satisfying to anyone interested in ethnic and social issues in archaeology, including the impact of colonization and the role played by different components of material culture in shaping early modern history in many parts of the world.

Apart from the very useful information, I find the most stimulating feature to be the strong direct or indirect emphasis on methodology and theory in archaeology and material culture studies. This contributes not only to a better understanding of our recent past but also to the development of archaeology as a whole. This is why the series gives real value at a very reasonable price. $\square$ Frans Verhaeghe is in the Department of Art History and Archaeology at the Free University of Brussels, Pleinlaan 2, B-1050 Brussels, Belgium.

\section{Free advice for experimenters}

\begin{tabular}{l}
\hline Technical Tips Online \\
Managing editor Mark Patterson \\
Elsevier. On-line only, free. \\
http://tto.trends.com \\
Laurie Goodman
\end{tabular}

Web-surfing scientists have a new reason to catch electronic waves. The fully - and solely - on-line journal Technical Tips Online provides quick, easy access to optimal methods for doing what researchers do best: experiments. Two great things about the site: it's free, and it's peer-reviewed. It therefore satisfies every web-surfer's belief that everything on the Internet should be free, and meets the need for high scientific standards for online-only resources.

Technical Tips Online is a fusion of a peerreviewed techniques journal and a methods database. It contains three types of article: peer-reviewed 'Technical Tips' (novel methods or major advances on known methods); non-peer-reviewed 'Protocols' (editorially invited protocols from laboratories with expertise in the method); and non-peerreviewed 'Application Notes' (companysubmitted paid-for reports detailing product use). Editorially selected comments from readers, which are quite fun to read, some with author responses, are also available.

The site is updated fortnightly and is extremely user-friendly. One can browse by category - Polymerase Chain Reaction, Electrophoresis, Microbiology, Purification Methods, Gene Expression, Microscopy, Tissue Culture, and Cloning and Sequencing - or examine only the latest additions. Using its comprehensive search engine is definitely superior to scanning several articles or journals for basic protocols, and then trying to glean modifications in later publications. After a while, however, this site could become equally unwieldy, because it might have to be searched just as extensively to obtain all useful protocol modifications. Given the ease of changing on-line text (which is what makes on-line protocols so appealing), this shouldn't be allowed to occur. Periodic processing of related information by the editors into a single update would increase this site's long-term value.

In this regard, it will be interesting to see how the site develops. Sustaining and properly maintaining any dedicated database is an enormous task, requiring time and money (it is supported by advertisements). All in all, however, if the site is well-maintained and the number of core protocols and useful modifications increases at a rapid rate, this site is well worth a bookmark in my book - er, computer.

Laurie Goodman is at Cold Spring Harbor Laboratory, 1 Bungtown Road, Cold Spring Harbor, New York 11724, USA.

\section{Fuel for thought}

\section{Combustion Theory and Modelling}

Editors-in-chief Bill Dold and Mitch

Smooke

Institute of Physics. 4/yr. USA \$257, elsewhere $£ 127$ (institutional); USA \$97, elsewhere $\mathfrak{E} 48$ (personal)

\section{A.C.Mclntosh}

Interest in combustion research has grown in recent years because of public pressure to find cleaner, more efficient means of energy production, which will all involve combustion of some kind for generations to come. 